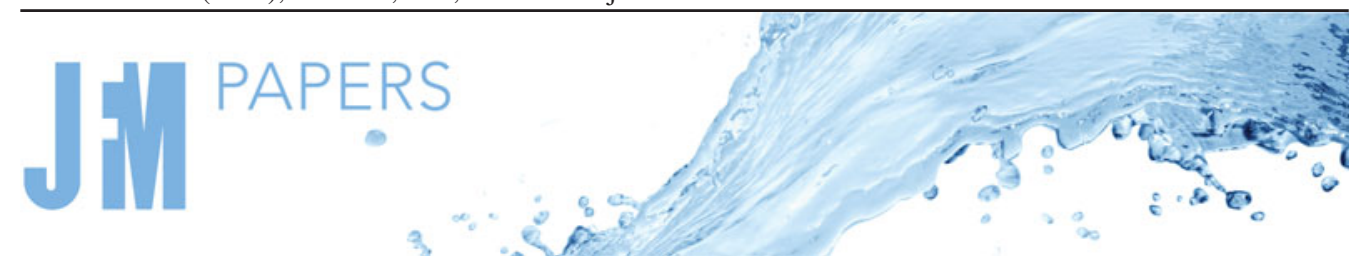

\title{
Acoustic flow in porous media
}

\author{
Ofer Manor $\dagger$ \\ Department of Chemical Engineering, Technion - Israel Institute of Technology, Haifa 32000, Israel
}

(Received 25 January 2021; revised 5 April 2021; accepted 10 May 2021)

We calculate the steady acoustic flow - the steady drift of fluid mass or acoustic streaming appearing along the path of an acoustic stimulus - in porous media. In particular, we suggest a mechanism to explain acoustic contributions to mass transport in porous media at geological, unit operation and lab-on-a-chip length scales. We study several cases of steady acoustic flow for a planar acoustic wave whose wavelength is large compared with the pore size. We commence our analysis at the ideal limit of same acoustic properties in the solid and fluid. The effective flow may then be treated intuitively according to the Darcy equation for flow through porous media in addition to a correction for the average azimuth of the pores compared with the acoustic path. We further consider the framework of a rigid porous frame, where the presence of a flow forcing mechanism resulting from the viscous dissipation of the acoustic wave at the solid surface of the pores hinders the intuitive application of the Darcy equation. However, we show that the steady acoustic flow in this case may be written as a quasi-Darcy-type equation. The analysis is conducted by a detailed calculation of the transport of mass through cylindrical pores of similar size but arbitrary azimuth compared with the acoustic path. We consider large, medium and small pore diameter limits relative to the viscous penetration length of the acoustic wave near the pore surface.

Key words: convection in porous media,microscale transport

\section{Introduction}

The transport of fluid in a porous medium under an acoustic stimulus is ubiquitous in geology and employed in unit operations and lab-on-a-chip platforms. For example, seismic waves appear to displace underground water (Elkhoury, Brodsky \& Agnew 2006); a high power acoustic excitation of $\mathrm{kHz}$ frequency appears to assist the motion of brine in sandstone and of air through packed beds of silica particles (Poesio et al. 2002;

$†$ Email address for correspondence: manoro@technion.ac.il

(C) The Author(s), 2021. Published by Cambridge University Press. This is an Open Access article, distributed under the terms of the Creative Commons Attribution licence (http://creativecommons.org/

licenses/by/4.0/), which permits unrestricted re-use, distribution, and reproduction in any medium, provided the original work is properly cited. 


\section{O. Manor}

Yao et al. 2012); the latter excitation further appears to separate underground oil/water mixtures (Hamida \& Babadagli 2008). The authors in the given studies have assumed different connections between the acoustic stimulus and transport of mass. In particular, the authors have suggested that the acoustic stimulus alters the soil structure or that the acoustic stimulus introduces thermal effects, which alter mass transport. However, these analyses are devoid of the possibility of a direct transport of momentum from the acoustic stimulus in the porous medium to a net flow of fluid therein.

A direct connection between acoustic excitation and flow through the instantaneous transport of momentum by convection is well known in the fields of ocean science and acoustic flow (acoustic streaming). The latter is the drift of mass appearing in the presence of acoustic waves. There are several types of acoustic flow mechanisms, which one may observe under acoustic excitation. Rayleigh (1884) examined flow patterns in Kundt tubes, where standing sound waves graze the tube surface to generate a periodic viscous boundary layer flow. The characteristic thickness of the boundary layer is referred to as the viscous penetration length of the acoustic wave in the fluid, $\delta=\sqrt{2 \mu / \rho \omega}$, where $\mu, \rho$ and $\omega$ are the shear viscosity and density of the fluid and the angular frequency of the acoustic wave, respectively. Rayleigh highlighted that weak convective effects support steady drift flow patterns - steady acoustic flow - which appear in the fluid beyond the boundary layer region. A similar acoustic boundary layer was observed and discussed previous to the work by Rayleigh (Chladni 1787; Faraday 1831) but without the use of mathematical tools to quantify the phenomenon. Schlichting (1932) extended the work by Rayleigh to predict the flow above a solid boundary which undergoes tangential vibrations. The weak convective nonlinearity in both problems gives rise to a similar expression for the drift flow far from the solid surface, which was coined the 'Rayleigh law of streaming' by Lighthill (1978) in his seminal review on this topic. We differentiate between the work by Lord Rayleigh and by Schlichting according to the type of excitation which they employed in their work. These are an acoustic wave in the fluid in the work by Lord Rayleigh and a vibration in the solid substrate in the work by Schlichting. In this paper, we are concerned with the former type of flow excitation. Hence, we refer to the resulting steady acoustic flow in the body of the paper in the presence or absence of a boundary layer flow by the notation: Rayleigh streaming.

The work by Lord Rayleigh and by Schlichting was further extended to account for the contribution of shallow ocean waves to drift flow by Longuet-Higgins (1953), for general periodic excitations in the fluid which are parallel to the solid boundary by Nyborg (1965), Nyborg (1952), Stuart (1966), Westervelt (2004) and Riley (1998, 2001), for periodic excitations by a combination of parallel and normal periodic motions of the solid boundary to account for the presence of propagating surface acoustic waves at a solid boundary under a half-space of fluid (Manor, Yeo \& Friend 2012; Morozov \& Manor 2016) and under a thin Landau-Levich-type coating film (Altshuler \& Manor 2015, 2016; Morozov \& Manor 2016), for acoustic flow inside channels (Hamilton, Ilinskii \& Zabolotskaya 2003) and for many other applications. Moreover, Eckart (1948) found that the same convective nonlinearity in the Navier-Stokes equations supports a steady drift in the form of vortical flows in the bulk of the fluid, far from any solid surface. The latter type of acoustic flow emerges as a result of variations in the intensity of an acoustic wave. Such variations appear to be due to the viscous and thermal attenuation of the waves in the bulk of the fluid (Shiokawa, Matsui \& Ueda 1989) or due to the spatial attenuation of an acoustic wave in the solid, which leaks (diffracts) planar acoustic waves (sound/ultrasound) into the fluid (Arzt \& Salzmann 1967; Campbell 1970). Kenneth, Keith \& Shawn (2004) and Reyta, Bailliet \& Valiere (2014) further used experiment and theory, respectively, 


\section{Acoustic flow in porous media}

to study acoustic streaming in cylindrical channels, a step toward realizing acoustic flow in cylindrical pores.

Late studies on the actuation of fluid by MHz frequency surface acoustic waves (SAWs) have in particular been used for generating phenomena that are known to be present during the transport of fluids in porous media. For example, SAWs have been used for manipulating (Miansari \& Friend 2016) and pumping (Guttenberg et al. 2004) liquids in nano-channels (Connacher et al. 2018; Xu 2018), powering dynamic wetting (Rezk et al. 2012, 2014; Altshuler \& Manor 2015; Manor et al. 2015; Horesh et al. 2019), manipulating thin liquid films in confined mixtures (Horesh, Morozov \& Manor 2017; Horesh, Zigelman \& Manor 2020) and actuating submicron thick electrolyte solutions for the analysis and manipulation of biochemical and biological agents (Friend \& Yeo 2011). Moreover, Ang et al. (2017) employed SAWs to pump water through membranes that were comprised of graphene nano-channels for eliminating nanometre size pollutants. Using a different platform, Martinez et al. (2010), Ho et al. (2011) and Parolo \& Merkoçi (2013) employed SAWs to pump water through porous paper channels, although it is believed that the flow mechanism in these cases is associated with SAW induced capillary effects in the paper channels. We now turn our attention to acoustic waves in porous media.

Many of the studies on acoustic waves in porous media were inspired by the work of Darcy (1856) on the linear connection between a pressure gradient, $\nabla p$, along the medium and the effective flow velocity, $\mathcal{U}=-(K / \mu) \nabla p$, through the pores, where $K$ is the permeability coefficient of the solid. In addition, the simplifying approach of modelling a porous medium as an array of channels in a solid medium is best known through the work of Kozney (1927) and Carman (1937, 1956), which connected the permeability of the solid to its porosity, $\zeta$. The Kozeny equation gives the single phase permeability $K=\beta \zeta^{3} * D_{p}^{2} /(1-\zeta)^{2}$, where $\beta$ and $D_{p}$ are an arbitrary proportionality coefficient and the characteristic length of pores in the solid, respectively. In the original study, the latter is meant to be the characteristic diameter of sand grains in a sand filter. Moreover, in the absence of inertia, the Ergun equation (Ergun 1952) gives a similar result for the permeability.

The first comprehensive studies on acoustic waves in porous media date back to Kirchhoff (1868), Rayleigh (1945) and to Zwikker \& Kosten (1949). The latter further integrated the former studies and additional studies given in references therein to elucidate the transport and absorption of sound waves in porous media of rigid and elastic frames with emphasis on thermal and viscous losses. In particular, early studies (Morse \& Bolt 1944; Scott 1946; Beranek 1947; Morse 1952) have predominantly concentrated on the transport of acoustic waves through the fluid phase within empty voids and porous media. They ignored, to a large extent, the transport of acoustic energy through the solid. This is the rigid frame approximation. This approximation is compatible with the case of a small acoustic particle motion in the solid relative to the one in the fluid and a large difference between the acoustic impedances of the two.

An extension to the rigid frame approximation (Beranek 1947) further attempted to account for the acoustic absorption of a randomly distributed solid matrix. It appears to agree with experiment in the case of soft fillings. However, rigid fillings render deviation of experiment from theory at acoustic frequencies above $1 \mathrm{kHz}$. Following studies further emphasized variable permeability and multiple fluid phases (Johnson 2001; Olny \& Boutin 2003; Pride \& Berryman 2003; Lo, Sposito \& Majer 2005), applications of sound propagation through porous biological media (Hosokawa \& Otani 1997; Haire \& Langton 1999) and an elastic porous frame, capable of supporting and scattering the propagation of acoustic wave modes. A partial list of the latter includes Zwikker \& Kosten (1949), Biot 


\section{O. Manor}

(1962), Attenborough (1971, 1982), Pride, Morgan \& Gangi (1993), Buckingham (1997), Zarek (2002), Williams et al. (2002), Sides (2003) and references therein. The transport of acoustic waves in both solid and fluid phases usually render the analysis complicated. The various details of the solid require different treatments and yield a large variety of corresponding theoretical models, which often require elaborate numerical procedures.

Our objective is to use previous studies on the propagation of an acoustic wave in a porous medium to find the resulting steady acoustic flow in the pores. A previous and highly technical study employed Green's function-type computations to assess the acoustic streaming in soft porous media during ultrasound measurements (Raghavan 2018). Our objective is to obtain analytical results to quantify acoustic streaming in simple porous media. We predominantly have in mind the case of $\mathrm{MHz}$ frequency acoustic waves, in line with modern developments in acoustofluidics. However, our analysis should be appropriate for lower acoustic frequencies as well. We restrict our attention to macro-scale acoustic effects by considering cases where the wavelength of sound is large compared with the pore size. The wave may be approximated as propagating in a homogenous medium of averaged acoustic properties, avoiding the necessity of accounting for acoustic diffraction resulting from the porosity of the solid. We consider several limits which yield analytical results for a steady acoustic flow in a porous medium. In the first case in $\S 2$, the acoustic impedance and phase velocity are same in the solid and fluid phases. We then consider the case of a rigid porous frame in $\S 3$, where the acoustic wave propagates solely through the fluid phase. In $\S 3.1$, we discuss previous work about the propagation of acoustic waves and then consider the steady acoustic flow in the limit where the pore size is large when compared with the viscous penetration length of the acoustic wave in $\S 3.2$ and in the limits where the pore size is comparable to or smaller with respect to the viscous penetration length in $\S 3.3$. We discuss and compare our results in $\S 4$ and conclude and summarize our findings in $\S 5$.

\section{Same acoustic properties in the fluid and solid}

Here, we assume that both the solid and fluid support the same acoustic properties - the same acoustic impedance and acoustic phase velocity - and that the fluid/solid interface is transparent to the acoustic wave since the latter does not differentiate between the solid and fluid phases. An implication of this assumption is that the leading-order displacement of molecules - the particle velocity of the acoustic wave - is the same in the fluid and solid. Hence, there is no acoustic boundary layer flow in the Rayleigh-type streaming near the solid/fluid interface at the pore surface. A difference between the motion of molecules in the solid and fluid phases does appear when considering secondary convective and viscous effects which bring about acoustic flow in the pores.

The leading-order component of the flow and pressure fields are associated with a propagating planar acoustic wave. The particle velocity of the wave is assumed to satisfy the form,

$$
u_{\text {wave }} \hat{\boldsymbol{x}}=U \cos (\omega t-k x) \mathrm{e}^{-\alpha x} \hat{\boldsymbol{x}},
$$

where $\hat{x}$ and $x$ are a unit vector and a coordinate along the path of the acoustic wave and $U, t, k, \omega$, and $\alpha$ are the particle velocity amplitude, time, wavenumber, angular frequency and attenuation coefficient, respectively. If one may postulate that the acoustic wave mostly undergoes viscous dissipation in the fluid phase of the porous medium, the attenuation coefficient may be approximated to leading order by a simple formula, $\alpha \approx \mu b k^{3} \zeta / 2 \rho \omega$, where $\mu b \equiv \mu_{b}+4 \mu / 3$ and $\mu_{b}$ is the bulk viscosity coefficient: This is the product of the attenuation coefficient in a pure fluid (Nyborg 1965) and the porosity of the solid, $\zeta$. 


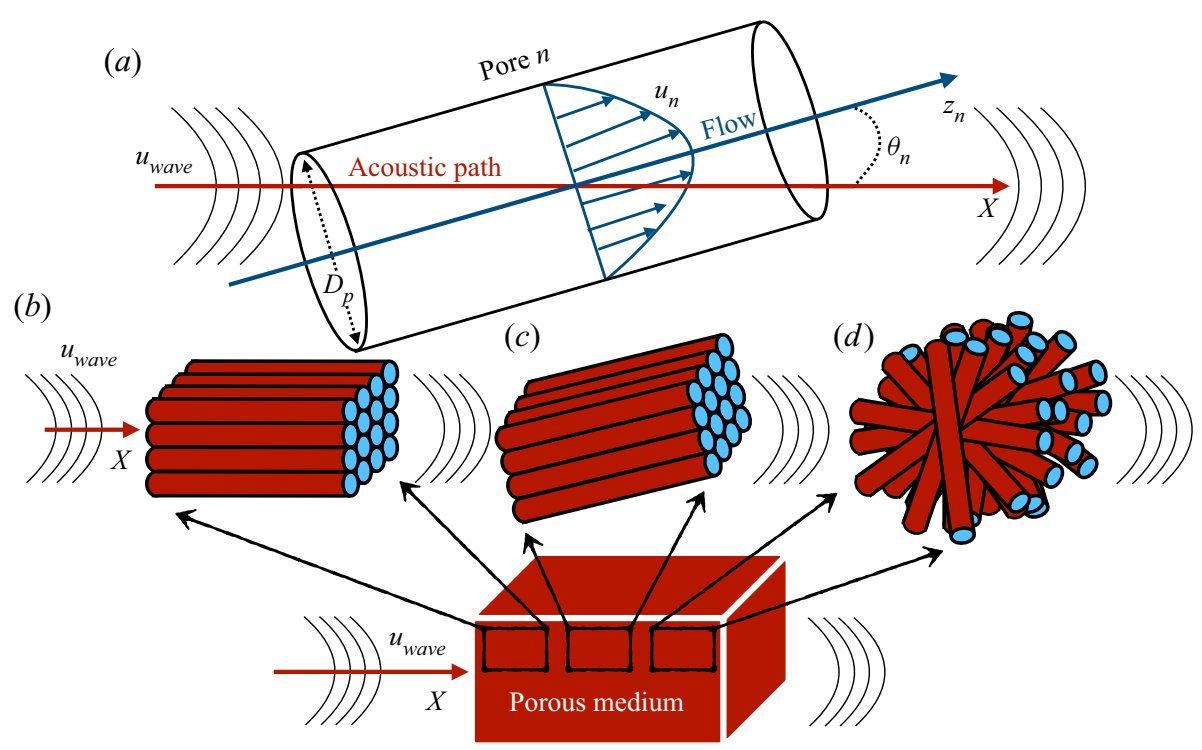

Figure 1. An illustration of a porous medium that is comprised of an array of cylindrical pores, where $(a)$ pore number $n$, of diameter $D_{p}$, is oriented so that its axial axis, $z_{n}$, and its axial flow component, $u_{n}$, are at an angle, $\theta_{n}$, with respect to the path of the acoustic wave, $x$; the pores in the solid medium may be $(b)$ aligned along the path of the acoustic wave, $(c)$ aligned along a path different to the acoustic wave or $(d)$ randomly aligned, among other options.

A previous analysis (Morse 1952) connected the particle velocities along the path of the acoustic wave, $x$, and its projection along the axial path of pore number $n$, given by $z_{n}$, using the relation $u_{n}=u_{\text {wave }} \hat{\boldsymbol{x}} \cdot \widehat{z_{\boldsymbol{n}}}=u_{\text {wave }} \cos \left(\theta_{n}\right)$. The terms $\hat{\boldsymbol{x}}$ and $\widehat{\boldsymbol{z}_{\boldsymbol{n}}}$ are unit vectors along the $x$ and $z_{n}$ coordinates and $\theta_{n}$ is the angle at which pore $n$ is aligned relatively to the path of the acoustic wave, respectively. This is illustrated in figure 1(a). Moreover, one may write the particle velocity along pore $n$ as,

$$
\boldsymbol{u}_{\boldsymbol{n}}=U \cos \left(\theta_{n}\right) \cos \left(\omega t-k z_{n}\right) \mathrm{e}^{-\alpha_{n} z_{n}} \widehat{z_{n}},
$$

where $\alpha_{n}$ is the rate of the wave attenuation along the coordinate $z_{n}$. Moreover, the assertion that at each point in space the particle velocity of the acoustic wave is unique requires that $\alpha x=\alpha_{n} z_{n}$.

As noted earlier, the same particle velocity across the fluid/solid boundary of the pore renders the absence of surface effects (acoustic boundary layer flow or Rayleigh streaming). As a consequence, the mechanism for acoustic flow in this case is Eckart streaming (Eckart 1948) in the bulk of the pore. Usually in such cases, the particle velocity is small compared with the phase velocity of the acoustic wave, so that $S t^{-1}=U k / \omega \ll 1$. The large Strouhal number, St, indicates that the contribution of viscous effects to the periodic flow along an acoustic wavelength is significant. One may thus represent the flow and pressure fields using the asymptotic series, $\boldsymbol{u}=\boldsymbol{u}_{0}(\boldsymbol{x}, t)+\boldsymbol{u}_{1}(\boldsymbol{x}, t)+\cdots ; p=$ $p_{0}(\boldsymbol{x}, t)+p_{1}(\boldsymbol{x}, t)+\cdots$, where $\boldsymbol{x}$ is an arbitrary position vector, $\left|\boldsymbol{u}_{0}\right| \gg\left|\boldsymbol{u}_{1}\right|$ and $\left|p_{0}\right| \gg$ $\left|p_{1}\right|$. In addition, $\boldsymbol{u} \equiv(u, v)$, where $u$ and $v$ are flow components along and transverse to the pore surface. Substituting the series in the Navier-Stokes equations yields a leading-order, $O(1)$, result, which is the particle velocity of the acoustic wave along the pore in (2.2), $u_{0}=u_{n}$. The second-order analysis, $O\left(S t^{-1}\right)$, has both periodic and steady components. Since we are interested in the steady component of the flow, we average over the equations 


\section{O. Manor}

using the operator $\langle Z\rangle \equiv \lim _{T \rightarrow \infty} \int_{t=0}^{T} Z \mathrm{~d} t$, where $Z$ and $T$ are an arbitrary function and a large time period such that periodic effects cancel each other, respectively. In the present problem, we assume an acoustic wave of one frequency. Thus, it is sufficient to require that $T=2 \pi / \omega$. The equations which govern the average steady acoustic flow through a pore are given by

$$
\left.\begin{array}{c}
\mu \nabla^{2}\langle\boldsymbol{u}\rangle_{1}-\nabla\langle p\rangle_{1}-F \hat{\boldsymbol{x}}=0, \\
F=\sum_{i=1}^{N} F_{n} \hat{\boldsymbol{x}} \cdot \widehat{z_{n}} / N=\sum_{n=1}^{N} F_{n} \cos \left(\theta_{n}\right) / N, \\
\equiv \rho\left\langle\boldsymbol{u}_{\boldsymbol{n}}(\boldsymbol{x}, t) \cdot \nabla \boldsymbol{u}_{\boldsymbol{n}}(\boldsymbol{x}, t)+\boldsymbol{u}_{\boldsymbol{n}}(\boldsymbol{x}, t) \nabla \cdot \boldsymbol{u}_{\boldsymbol{n}}(\boldsymbol{x}, t)\right\rangle,
\end{array}\right\}
$$

where $N$ is an arbitrary (large) number of cylindrically shaped pores in a unit volume. The first equation in (2.3) governs the average flow per pore along the path of the acoustic wave, $\hat{x}$; the second expression gives the spatially averaged acoustic forcing, $F$, to a steady acoustic flow along the same path; the third expression gives the acoustic forcing, $F_{n}$, to a steady acoustic flow along pore $n$ (along $\widehat{z_{n}}$ ).

Substituting (2.2) in (2.3) gives that the time averaged forcing term along a pore is,

$$
F_{n}=2 \rho\left\langle u_{n} \times \mathrm{d} u_{n} / \mathrm{d} z_{n}\right\rangle=-2 \rho \alpha_{n} U^{2} \mathrm{e}^{-2 \alpha_{n} z_{n}} \cos ^{2}\left(\theta_{n}\right)
$$

The spatially averaged acoustic forcing per pore along the path of the acoustic wave, $\hat{x}$, where each pore may be arranged arbitrarily with respect to the path of the wave at an angle $\theta_{n}$, is given by,

$$
F=\sum_{n=1}^{N} \cos \left(\theta_{n}\right) F_{n} / N=-2 \rho \alpha U^{2} \mathrm{e}^{-2 \alpha x} \sum_{i=1}^{N} \cos ^{2}\left(\theta_{n}\right) / N=-2 \rho \alpha U^{2} \mathrm{e}^{-2 \alpha x} / m
$$

where we employed the equality $\alpha_{n} z_{n}=\alpha x$ and the connection $x=z_{n} \cos \left(\theta_{n}\right)$ which requires that $\alpha=\alpha_{n} / \cos \left(\theta_{n}\right)$. The term $m=N / \sum_{i=1}^{N} \cos ^{2}\left(\theta_{n}\right)$ is the structural coefficient of the porous medium (Morse 1952). In the case that the pores are aligned along $\left(\theta_{n}=0\right)$ or perpendicular to $\left(\theta_{n}=\pi / 2\right)$ the propagating acoustic wave, we obtain that $m=1$ or $\infty$, respectively; see an illustration in figure $1(b, c)$. Another case is randomly oriented pores. The pores are arbitrarily aligned between the two limiting cases of the angles $\theta_{n}=0$ and $\pi / 2$; see an illustration in figure $1(d)$. In this case, one may consider an arbitrary volume which contains a large number of pores, to find that $\sum_{i=1}^{N} \cos ^{2}\left(\theta_{n}\right) / N \approx$ $(1 / V) \int_{V} \cos ^{2}(\theta) d V=1 / 3$, where $\theta=[0, \pi / 2]$ is the smoothed (or coarse-grained) angle between the pores and the acoustic wave. The corresponding magnitude of the structural coefficient in this case is $m=3$.

Substituting (2.5) in (2.3) gives an equation for the average momentum conservation in a pore along the path of the acoustic wave. By further accounting for the porosity, $\zeta$, we may obtain the effective flow in a porous medium. However, in this case, we avoid these technicalities and further restricting assumptions about the structure of the porous medium or specific boundary conditions for the flow at the surface of the pores. We take advantage of the representation of the acoustic forcing term, $F$, in a form which is similar to the pressure gradient forcing term in the Darcy equation to write the effective flow rate 
through a porous medium,

$$
\mathcal{U}=-\frac{K}{\mu}\left(F+\frac{\partial\langle p\rangle_{1}}{\partial x}\right)=\frac{2 \rho \alpha K U^{2}}{m \mu} \mathrm{e}^{-2 \alpha x},
$$

where, following the second equality, we assume that the steady pressure in the pores is the ambient pressure and thus, $\partial\langle p\rangle_{1} / \partial x=0$. Moreover, the permeability coefficient, $K$, naturally accounts for the porosity, size and shape distribution of pores, and boundary conditions at the fluid/solid interface. The similarity between the Darcy equation and (2.6) suggests that one may obtain the magnitude of $K$ from one type of flow excitation to study the volume flux under another type of excitation.

The assumption that the pressure in the porous medium is ambient, $\partial\langle p\rangle_{1} / \partial x=0$, which we follow throughout this work, is appropriate in the case in which the width of the acoustic wave front is smaller than the width of the porous medium of interconnected pores. In this case, fluid in the porous medium is free to flow between regions which are excited by and devoid of the acoustic wave to conserve mass and alleviate pressure differences. Ignoring viscous dissipation in the motion of the fluid between these regions renders the pressure throughout the porous medium the ambient pressure. A different limit is the case in which the width of the acoustic wave is at least as large as the width of the porous medium. The latter is closed to fluid flow on the sides. Thus, the fluid may not flow to regions which are devoid of the acoustic wave. The attenuation of the acoustic wave will result in a spatial variation in the pressure in the porous medium, $\langle p\rangle_{1}$, which will satisfy the mass conservation of fluid by imposing additional Poiseuille flow in the pores. Assuming an ambient pressure at the entrance of fluid to the porous medium and that the intensity of the acoustic wave does not change normal to its path (a flat front of the acoustic wave), the contribution of the spatially varying pressure, $\langle p\rangle_{1}$, will exactly cancel the contribution of the acoustic attenuation to fluid flow along the acoustic path. This will result in a similar expression for the effective velocity of fluid as in (2.6), albeit in the absence of the attenuation term: $\mathcal{U}=2 \rho \alpha K U^{2} / m \mu$. Similar arguments apply in the following case of a rigid porous frame.

\section{Rigid porous frame}

\subsection{Acoustic wave}

In this section we assume a large difference between the acoustic impedance in the solid and fluid phases. The acoustic wave exists predominantly in the fluid. We further assume that the wavelength of the acoustic wave is large compared with the diameter of the pores, $k D_{p} \ll 1$, and could be taken as propagating in a medium of averaged acoustic impedance. In our analysis, we will employ the seminal work on the propagation of sound waves in rigid porous media by Morse (1952). The simple results and insights given by Morse appear to agree well with experiment. In particular, our approach is compatible with their guiding assumption that $k D_{p} \ll 1$. Moreover, they assume that the pores are cylindrical tubes and are arbitrarily aligned, in a similar manner to the structural assumptions by Kozney (1927) and Carman (1937, 1956) for the structure of porous media. Under these assumptions, a propagating wave in a rigid porous medium may be described by (2.1) and (2.2). The wavenumber is given by $k=\omega \sqrt{m} / c_{0}$, the attention coefficient is given by $\alpha=\mathcal{R} \zeta /\left(2 \rho c_{0} \sqrt{m}\right)$ and the effective phase velocity is $c=c_{0} / \sqrt{m}$, where $c_{0}$ is the phase velocity of the acoustic wave in the fluid and $\mathcal{R}$ is the dynamic flow resistance in the pores.

One may distinguish two regimes (Zwikker \& Kosten 1949), which are determined by the ratio between the diameter of the pores, $D_{p}$, and the viscous penetration length, 


\section{O. Manor}

$\delta=\sqrt{2 \mu / \rho \omega}$. In the case that $D_{p} \ll \delta$, one may approximate the attenuation of the acoustic wave by assuming a viscous Poiseuille flow in the pores, which gives $\mathcal{R} \approx$ $8 \mu \mathrm{m} / \zeta D_{p}^{2}$. In the opposite case, where $D_{p} \gg \delta$, one may approximate the leading-order attenuation of the acoustic wave by accounting for the dynamic convection of momentum and viscous dissipation in the pores, which gives $\mathcal{R} \approx m \sqrt{2 \mu \omega \rho} / \zeta q D_{p}$; the property $q$ is a proportionality coefficient between the size of the pore under acoustic excitation and at rest.

The two regimes, discussed in the previous paragraph, mark two regimes of acoustic flow. One regime is associated with a large pore size, $D_{p} \gg \delta$. Hence, one expects a boundary layer flow (Rayleigh streaming) near the solid boundary of a pore, where inertial and viscous contributions to the flow are comparable. In addition, one may expect a unidirectional bulk flow away from the pore surface. Both the viscous dissipation of the acoustic wave in the bulk (Eckart 1948), known as Eckart streaming, and near the solid surface of the pore (Rayleigh 1884), known as Rayleigh streaming, contribute to the acoustic flow. The second regime is associated with a medium to small pore size, $D_{p}<\delta$. The contribution to the flow in this case is mainly associated with a Rayleigh-type streaming. The acoustic flow appears due to the viscous dissipation of the acoustic wave near the solid boundary of the pore, albeit the pore is not wide enough to support a boundary layer type flow.

\subsection{Acoustic flow in large pores $\left(\delta \ll D_{p} \ll k^{-1}\right)$}

When the diameter of the pore, $D_{p}$, is large compared with the viscous penetration length, $\delta$, one may consider the contribution of bulk and surface mechanisms to the overall acoustic flow in a pore. By integrating both flow mechanisms and the structural properties of the porous medium, we obtain the effective volume flux through the medium.

\subsubsection{Acoustic flow near the surface of the pore - Rayleigh streaming}

In this case, where the characteristic thickness of the boundary layer flow is small when compared with the diameter of the pores, $\delta \ll D_{p}$, one may ignore the contribution of the radial curvature at a pore surface to the boundary layer flow. One may assume to leading order a problem of flow near a flat surface. Examples of previous studies on acoustic boundary layer flow near flat surfaces are standing acoustic waves in the absence of acoustic attenuation (Rayleigh 1884; Stuart 1966), standing and propagating ocean waves in the absence of wave attenuation (Longuet-Higgins 1953), standing wave motion in the solid, tangent to the solid surface, (Schlichting 1932) and propagating SAWs in a solid substrate that possesses both tangent and transverse motion components (Manor et al. 2012; Morozov \& Manor 2016).

Below, we solve for the acoustic flow due to the presence of an acoustic wave in the fluid, which grazes a flat solid surface. Unlike most studies, we account for the attenuation of the propagating wave in our calculations. In $\S 3.1$ we highlight that the rate of wave attenuation in a rigid porous frame may be faster than in a pure fluid. Hence, one must examine the possibility of greater non-local contributions than usual from the attenuation of the wave to the acoustic flow. However, we show that even in this case, the non-local contribution of the attenuation coefficient to the acoustic flow near a solid surface is small. Previous descriptions of the contribution of acoustic attenuation to flow in channels are given elsewhere (Doinikov, Thibault \& Marmottant 2017; Pavlic \& Dual 2021).

We ignore the curvature of the pore $\left(\delta \ll D_{p}\right)$ in the boundary layer and adopt Cartesian coordinates, $z_{n}$ and $y=D_{p} / 2-r$, along pore $n$ and transverse to the pore 


\section{Acoustic flow in porous media}

surface, respectively. Outside the boundary layer flow, the leading-order velocity field is given by the acoustic wave in (2.2). Moreover, the typical thickness of the pores under consideration is assumed small compared with the acoustic wavelength, $D_{p} \ll k^{-1}$, which renders compressional contributions to flow in a pore small (Rayleigh 1884; Stuart 1966). To calculate the acoustic flow in a pore, we rewrite the continuity and Navier-Stokes equations in terms of the streamfunction $\psi$. The latter is defined by using the axial and radial components of the velocity field $(u, v)=\left(\psi_{y},-\psi_{z_{n}}\right)$, respectively, along pore $n$. In what comes next, we use a shorthand notation for derivatives, e.g. $\psi_{z_{n}} \equiv \partial \psi / \partial z_{n}$ and $\psi_{z z} \equiv \partial^{2} \psi / \partial z_{n}^{2}$, alongside the usual derivative notation.

Using the scaling transformations,

$$
t \rightarrow t / \omega, \quad y \rightarrow \delta y, \quad z_{n} \rightarrow z_{n} / k, \quad \psi \rightarrow \delta U \psi, \quad \alpha_{n} \rightarrow k \alpha_{n},
$$

we write the dimensionless streamfunction equation and boundary conditions in Cartesian coordinates,

$$
\left.\begin{array}{c}
2 \psi_{y y t}+S t^{-1}\left(\psi_{y} \psi_{z_{n} y y}-\psi_{z_{n}} \psi_{y y y}\right)=\psi_{y y y y}+\cdots, \\
\left.(u, v)\right|_{r=0}=(0,0),\left.u\right|_{r \rightarrow \infty}=\cos \left(\theta_{n}\right) \cos \left(t-z_{n}\right) \mathrm{e}^{-\alpha_{n} z_{n}},
\end{array}\right\}
$$

where we require the velocity field far from the solid surface to satisfy the particle velocity of the acoustic wave in the bulk of the pore, (2.2); we assume that the fluid satisfies a no-slip condition at the pore surface and does not penetrate the solid; the reciprocal of the Strouhal number, $S t^{-1}$, is a small number, and the term '. .' ' is associated with additional small contributions to the equation, which are multiplied by powers of $\delta k$. Expanding the streamfunction in $S t^{-1}, \psi=\psi_{0}+S t^{-1} \psi_{1}+\cdots$, gives the leading-order problem,

$$
2 \psi_{0, y y t}=\psi_{0, y y y y},\left.\quad\left(\psi_{0, y},-\psi_{0, x}\right)\right|_{r=0}=(0,0), \quad \psi_{0, y}=\cos \left(\theta_{n}\right) \cos \left(t-z_{n}\right) \mathrm{e}^{-\alpha_{n} z_{n}},
$$

which is satisfied by the solution,

$$
\begin{aligned}
\psi_{0}= & \cos \left(\theta_{n}\right) \mathrm{e}^{-\alpha_{n} z_{n}-y}\left(\sin \left(t-z_{n}-y\right)+\cos \left(t-z_{n}-y\right)\right) / 2 \\
& \left.+\cos \left(\theta_{n}\right) \mathrm{e}^{-\alpha_{n} z_{n}}\left((2 y-1) \cos \left(t-z_{n}\right)-\sin \left(t-z_{n}\right)\right)\right) / 2+f(t),
\end{aligned}
$$

where $f(t)$ is a function of time, which does not contribute to the velocity field. We demonstrate the streamlines of the solution in figure 2 .

We are interested in the steady component of the solution. Hence, we average the second-order problem over long times using the operator \langle\rangle , which we discuss in $\S 2$. The leading-order, time averaged, problem takes then the form,

$$
\left.\begin{array}{c}
2\langle\psi\rangle_{1, y y t}-\langle\psi\rangle_{1, y y y y}=-\left\langle\psi_{0, y} \psi_{0, z_{n} y y}-\psi_{0, z_{n}} \psi_{0, y y y}\right\rangle, \\
\left.\left(\langle\psi\rangle_{1, y},-\langle\psi\rangle_{1, x}\right)\right|_{r=0}=(0,0),
\end{array}\right\}
$$

in addition to the relaxed requirement that the second-order component of the solution $\psi_{1}$ is not more singular than the leading-order solution $\psi_{0}$. The problem is satisfied by the solution,

$$
\begin{aligned}
\langle\psi\rangle_{1}= & \cos ^{2}\left(\theta_{n}\right) \mathrm{e}^{-2 \alpha_{n} z_{n}}\left[\left(-13 \alpha_{n}+6\left(\alpha_{n}+1\right) y-3\right) / 8+\left(\alpha_{n}-1\right) \mathrm{e}^{-2 y} / 8\right. \\
& \left.+\cos ^{2}\left(\theta_{n}\right) \mathrm{e}^{-y}\left(\left(\alpha_{n}(r+2)-2\right) \sin (y)+\left(3 \alpha_{n}+y+1\right) \cos (y)\right) / 2\right] .
\end{aligned}
$$

We plot the axial component of the flow field for different magnitudes of $\alpha_{n}$ in figure 3 . The axial acoustic flow far away from the solid surface of the pore is then given by 


\section{O. Manor}

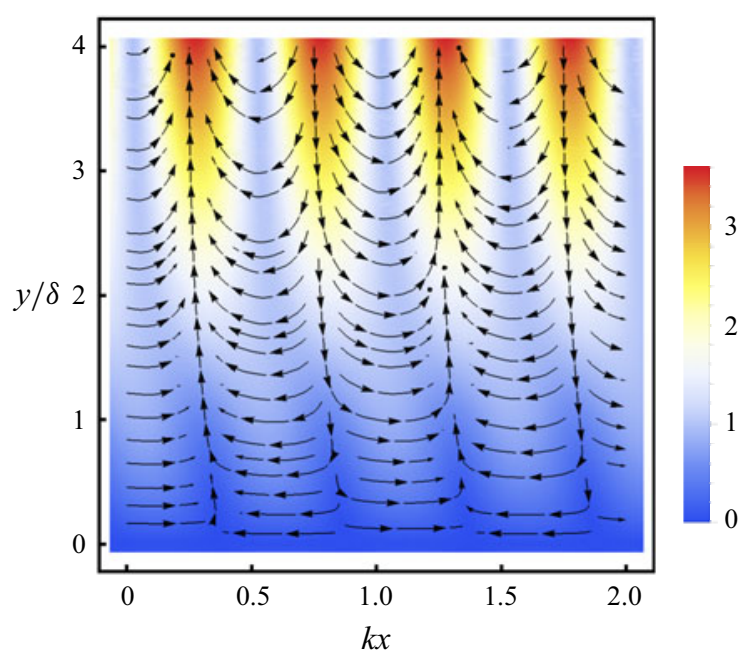

Figure 2. Spatial variations of the velocity field, given by the scaled streamfunction $\psi_{0} / \delta U \cos \left(\theta_{n}\right)$ along the axial and radial coordinates $k x$ and $y / \delta$, respectively, where we ignore wave attenuation $\left(\alpha_{n}=0\right)$ and time, define the surface of the pore at $y=0$ and represent the different properties using dimensional notation. We use arrows to give the path of the velocity field and colours to indicate the relative flow velocity, which is quantified in the colour legend to the right.

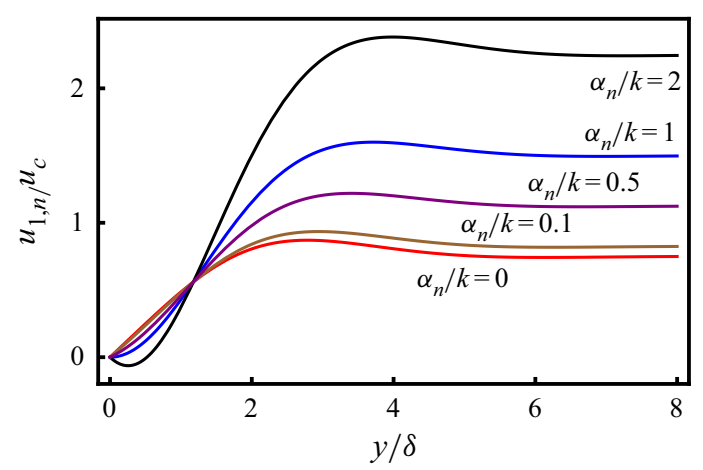

Figure 3. Spatial variations of the dimensional axial drift velocity, $u_{n}$, along pore $n$ for different values of the effective wave attenuation coefficient along the pore $\alpha_{n}$, where $u_{c} \equiv S t^{-1} \cos ^{2}\left(\theta_{n}\right) \mathrm{e}^{-2 \alpha_{n} z_{n}} U$ and where we represent the different properties using dimensional notation. The acoustic streaming far from the solid, $u_{d, n}=$ $u_{n}(y / \delta \gg 1)$, flows along the path of the wave and increases in magnitude with the attenuation coefficient, $\alpha_{n}$, although the acoustic flow may change direction near the solid surface when $\alpha_{n} k>1$.

$u_{d, n}=\lim _{y \rightarrow \infty}\langle\psi\rangle_{y}=S t^{-1} \lim _{y \rightarrow 0}\langle\psi\rangle_{1, y}$, noting that $\psi_{0}$ is a periodic function of time and hence vanishes over long times. Introducing (3.6) in the expression for $u_{d, n}$, gives that,

$$
u_{d, n}=3 S t^{-1} \cos ^{2}\left(\theta_{n}\right) \mathrm{e}^{-2 \alpha_{n} z_{n}}\left(1+\alpha_{n}\right) / 4 .
$$

The first term in the brackets appears from the contribution of the interaction between the solid surface and the acoustic wave. The second term is the non-local contribution of the wave attenuation along the pore. The dimensional counterpart of (3.7) is given by,

$$
u_{d, n}=3 S t^{-1} U \cos ^{2}\left(\theta_{n}\right) \mathrm{e}^{-2 \alpha_{n} z_{n}}\left(1+\alpha_{n} / k\right) / 4 .
$$




\section{Acoustic flow in porous media}

Moreover, the radial component of the acoustic flow, $v_{d, n}=\lim _{y \rightarrow \infty}\left\langle-\psi_{z_{n}}\right\rangle=O\left(S t^{-1} \delta k\right)$, is small since $\delta k \ll 1$.

\subsubsection{Acoustic flow in the bulk of the pore}

The flow in the bulk of the pore is of a characteristic radial length, $D_{p}$, which is large compared with the viscous penetration length, $\delta$, so that $D_{p} \gg \delta$. Hence, the axial drift flow, $u_{d, n}$, in (3.8) is a boundary condition of magnitude $O\left(U S t^{-1}\right)$ to the bulk acoustic flow near the pore surface. This analysis is equivalent to using the leading-order term of a matched asymptotic expansion procedure to connect between the acoustic flow in the boundary layer and in the bulk of the pore.

In a similar manner to the analysis leading to (2.3), the mathematical description of the unidirectional flow along the axial path, $z_{n}$, of pore $n$, away from the pore surface, is given by representing the axial flow and pressure field in the porous medium using the asymptotic series, $\boldsymbol{u}=\boldsymbol{u}_{0}(\boldsymbol{x}, t)+\boldsymbol{u}_{1}(\boldsymbol{x}, t)+\cdots ; p=p_{0}(\boldsymbol{x}, t)+p_{1}(\boldsymbol{x}, t)+\cdots$, where $\left|\boldsymbol{u}_{0}\right| \gg\left|\boldsymbol{u}_{1}\right|$ and $\left|p_{0}\right| \gg\left|p_{1}\right|$. As before, $\boldsymbol{u}=(u, v)$, where the former and latter flow components are along and transverse to the pore. Substituting the series in the Navier-Stokes equations gives the leading-order periodic flow velocity along the pore in (2.2), namely $u_{0}=u_{n}$. It is the component of the acoustic wave along pore $n$. The leading steady flow in the same pore is given by time averaging over the equations and boundary conditions, which yields,

$$
\mu \nabla^{2}\langle\boldsymbol{u}\rangle_{1}-\nabla\langle p\rangle_{1}-F_{n} \widehat{z_{\boldsymbol{n}}}=0,
$$

where $F_{n}$ is given in (2.4). Moreover, in a pore, the radial coordinate, $r$, scales like the pore diameter, $D_{p}$, and the axial coordinate, $z_{n}$, scales like the wavenumber $k^{-1}$. Considering that $D_{p} \ll k^{-1}$, the problem in (3.9) may be simplified to

$$
\mu \frac{1}{r} \frac{\mathrm{d}}{\mathrm{d} r}\left(r \frac{\mathrm{d}\langle u\rangle_{1}}{\mathrm{~d} r}\right)-\frac{\partial\langle p\rangle_{1}}{\partial z_{n}}-F_{n}+\cdots=0,
$$

where we require radial symmetry of the flow about the centre line of the pore (along $\widehat{z_{n}}$ ). The flow field should further satisfy the boundary conditions

$$
\mathrm{d}\langle u\rangle_{1} /\left.\mathrm{d} r\right|_{r=0}=0,\left.\quad\langle u\rangle_{1}\right|_{r \rightarrow D_{p} / 2}=u_{d, n},
$$

where $u_{d, n}$ is given in (3.8). The problem in $(3.10)$ and $(3.11 a, b)$ is satisfied by the steady flow in the pore

$$
\langle u\rangle_{1}=\cos ^{2}\left(\theta_{n}\right) U^{2} \mathrm{e}^{-2 \alpha_{n} z_{n}}\left(\frac{12 \alpha_{n} \mu+\alpha_{n} D_{p}^{2} \rho \omega+12 k \mu}{16 \mu \omega}-\frac{\alpha_{n} \rho r^{2}}{4 \mu}\right),
$$

where we assume the absence of a steady pressure distribution $\partial\langle p\rangle_{1} / \partial z_{n}=0$. The average flow along the pore is then

$$
u_{\text {pore }, n}=A^{-1} \int_{A}\langle u\rangle_{1} \mathrm{~d} A=\cos ^{2}\left(\theta_{n}\right) U \mathrm{e}^{-2 \alpha_{n} z_{n}}\left(\frac{R e}{32} D_{p} \alpha_{n}+\frac{3 S t^{-1}}{4}\left(1+\frac{\alpha_{n}}{k}\right)\right),
$$

where $A=\pi D_{p}^{2} / 4$ is the cross-section (radial) area of the cylindrical pore, $\mathrm{d} A=2 \pi r \mathrm{~d} r$, and $\operatorname{Re} \equiv \rho U D_{p} / \mu$. In (3.13), we neglect the volume flux inside the boundary layer region. The first and the second terms in the outer brackets are contributions to the flow in the bulk 


\section{O. Manor}

of the pore resulting from the attenuation of the acoustic wave and the acoustic boundary layer flow (Rayleigh streaming). Moreover, we average the flow rate along the path of the wave over a large number of pores, $N$, where each pore may be aligned arbitrarily with respect to the wave path at an angle, $\theta_{n}$. By further accounting for the porosity $\zeta$ of the solid, we obtain the effective steady flow rate through the integral porous medium along the path of the acoustic wave, $\hat{\boldsymbol{x}}$,

$$
\mathcal{U}=\frac{\zeta}{N} \sum_{n=1}^{N} \cos \left(\theta_{n}\right) u_{\text {pore }, n}=U \zeta \mathrm{e}^{-2 \alpha x}\left(\frac{D_{p} \alpha}{32} \frac{R e}{m}+\frac{3}{4}\left(1+\frac{m^{\prime}}{m} \frac{\alpha}{k}\right) \frac{S t^{-1}}{m^{\prime}}\right),
$$

where we employ the equality $\alpha_{n} z_{n}=\alpha x$, the connections $x=z_{n} \cos \left(\theta_{n}\right)$ and $\alpha=$ $\alpha_{n} / \cos \left(\theta_{n}\right)$, the definition of the structural coefficient $m=N / \sum_{n=1}^{N} \cos ^{2}\left(\theta_{n}\right)$ and define the modified structural coefficient $m^{\prime}=N / \sum_{n=1}^{N} \cos ^{3}\left(\theta_{n}\right)$. The analysis is similar to the one that yields (2.5) and is discussed in $\S 2$. Moreover, in the case that the pores are aligned along or perpendicular to the propagating acoustic wave, we obtain that $m^{\prime}=1$ and $\infty$, respectively, in a similar manner to $m$. In the case of randomly oriented pores, where the pores may be aligned between the two limiting cases of $\theta_{n}=0$ and $\pi / 2$, we obtain that $m^{\prime}=4$, noting again that $m=3$ in this case.

As a last note in this section, we assess the contribution of the attenuation coefficient, $\alpha$, to the effective acoustic flow in the porous medium, $\mathcal{U}$. We find that the term for the non-local attenuation contribution to the flow near the solid surface of the pores, $\left(m^{\prime} / m\right)(\alpha / k)$ in the inner brackets in (3.14), is small. Briefly, the attenuation coefficient may be approximated by $\alpha=R \zeta /(2 \rho \mathrm{cm})$, where $\mathcal{R} \approx m \sqrt{2 \mu \omega \rho} / \zeta q D_{p}$. See our discussion in $\S 2.1$ for further detail. We substitute the approximations for $\alpha$ and $\mathcal{R}$ in (3.14) and obtain that $\alpha / k=(2 q)^{-1}\left(\delta / D_{p}\right)$, where we employ the relation $c=\omega / k$. The relative deviation of the pore size under acoustic excitation from its size at rest, $q$, should be near unity. Hence, it is apparent that $\alpha / k \ll 1$ since $\delta / D_{p} \ll 1$. In addition, the terms $m$ and $m^{\prime}$ are similar in magnitude, so that $m^{\prime} / m=O(1)$. It is not a surprise that the non-local contribution of the wave attenuation to the acoustic flow near the solid surface of the pore is small. Most studies on acoustic flow near a solid surface neglect non-local contributions from the attenuation of the acoustic wave. However, most studies usually consider the presence of the acoustic wave in a half-space of fluid, where the rate of acoustic attenuation is slower than in the present case. Thus, this insight for the case of a porous medium is not trivial. In conclusion, one may approximate the effective steady flow rate by

$$
\mathcal{U} \approx U \zeta \mathrm{e}^{-2 \alpha x}\left(\frac{D_{p} \alpha}{32} \frac{R e}{m}+\frac{3}{4} \frac{S t^{-1}}{m^{\prime}}\right)
$$

\subsection{Acoustic flow in small pores $\left(D_{p}<\delta \ll k^{-1}\right)$}

\subsubsection{Problem definition}

As noted previously in $\S 3.2$, the typical thickness of the pores under consideration is assumed small compared with the acoustic wavelength, $D_{p} \ll k^{-1}$, which renders compressional contributions to flow in a pore small (Rayleigh 1884; Stuart 1966). As before, we calculate the acoustic flow in a pore by rewriting the continuity and Navier-Stokes equations in terms of the streamfunction $\psi$. The streamfunction in this case is associated with cylindrical coordinates and satisfies that $(u, v)=\left(\psi_{r} / r,-\psi_{z_{n}} / r\right)$. 


\section{Acoustic flow in porous media}

Using the scaling transformations,

$$
t \rightarrow t / \omega, \quad r \rightarrow \delta r, \quad \alpha_{n} \rightarrow \alpha_{n} k, \quad z_{n} \rightarrow z_{n} k^{-1}, \quad \psi \rightarrow \delta^{2} U \psi
$$

we write the dimensionless streamfunction equation (Leal 2007)

$$
\begin{aligned}
& 2 \frac{\psi_{t r}}{r}-2 \psi_{t r r}-3 \frac{\psi_{r}}{r^{3}}+3 \frac{\psi_{r r}}{r^{2}}-2 \frac{\psi_{r r r}}{r}+\psi_{r r r r} \\
& \quad+S t^{-1}\left(3 \frac{\psi_{z_{n}} \psi_{r}}{r^{3}}+\frac{\psi_{r} \psi_{r z_{n}}}{r^{2}}-3 \frac{\psi_{z_{n}} \psi_{r r}}{r^{2}}-\frac{\psi_{r} \psi_{r r z_{n}}}{r}+\frac{\psi_{z_{n}} \psi_{r r r}}{r}\right)+\cdots=0,
\end{aligned}
$$

where $S t^{-1} \ll 1$ and where we omit smaller-order terms which are multiplied by powers of $\delta k$. We require a no-slip condition at the pore surface, $\left.u\right|_{r=R}=0$, and that the velocity field satisfies symmetry at the centre of the pore, $\left.\left(u_{r}, v\right)\right|_{r=0}=(0,0)$. Moreover, we employ an additional constraint by following the guidelines of the analysis by Zwikker \& Kosten (1949) in their attempt to calculate the viscous dissipation of an acoustic wave, which propagates through small pores. We require that the leading-order solution to (3.17) satisfies a volume flux in pore $n$, which is comparable to the one generated by the acoustic wave, $\int_{A} u \mathrm{~d} A=A \cos \theta_{n} \cos \left(t-z_{n}\right) \mathrm{e}^{-\alpha_{n} z_{n}}+\cdots$, where $A=\pi D_{p}^{2} / 4$ is the (radial) cross-sectional area of the cylindrical pore, $\mathrm{d} A=2 \pi r \mathrm{~d} r$, and the term ' ...' is associated with smaller-order corrections. The steady component of the correction to the flow in the latter is our goal in this analysis. Moreover, the conservation of fluid mass requires finite periodic radial velocity at the surface of the pore. Hence, the pore radius, $R$, is not a constant but varies in time and space. It is given by the kinematic condition

$$
R_{t}=S t^{-1} v
$$

subject to a no-slip condition at the pore surface.

To simplify the analysis of the problem in (3.17), (3.18) and the given boundary conditions, we expand the streamfunction, corresponding velocity field and the radius of the pore in the inverse of the Strouhal number, $S t^{-1}$, by assuming the series

$$
\left.\begin{array}{rl}
\psi & =\psi_{0}+S t^{-1} \psi_{1}+\cdots, \\
R & =R_{0}+S t^{-1} R_{1}+\cdots \cdot \\
u & =u_{0}+S t^{-1} u_{1}+\cdots, \\
v & =v_{0}+S t^{-1} v_{1}+\cdots,
\end{array}\right\}
$$

where $R_{0} \equiv D_{p} / 2$ is the radius of the pore at rest.

\subsubsection{Leading-order problem}

Substituting the series in (3.19) in the streamfunction equation in (3.17) and in the corresponding boundary conditions gives the leading-order problem, $O(1)$

$$
\left.\begin{array}{c}
2 \frac{\psi_{0, t r}}{r}-2 \psi_{0, t r r}-3 \frac{\psi_{0, r}}{r^{3}}+3 \frac{\psi_{0, r r}}{r^{2}}-2 \frac{\psi_{0, r r r}}{r}+\psi_{0, r r r r}=0, \\
\left.u_{0}\right|_{r=R_{0}}=\left.u_{0, r}\right|_{r=0}=\left.v_{0}\right|_{r=0}=0, \quad 2 \int_{r=0}^{R_{0}} u_{0} r \mathrm{~d} r=R_{0}^{2} \cos \left(\theta_{n}\right) \cos \left(t-z_{n}\right) \mathrm{e}^{-\alpha_{n} z_{n}} .
\end{array}\right\}
$$

The solution of the problem is simplified by assuming that $\psi_{0}=\operatorname{Real}\left\{g(r) \mathrm{e}^{i\left(t-z_{n}\right)-\alpha_{n} z_{n}}\right\}$, where $g(r)$ is a complex function of $r$. Further accounting for the boundary conditions in 


\section{O. Manor}

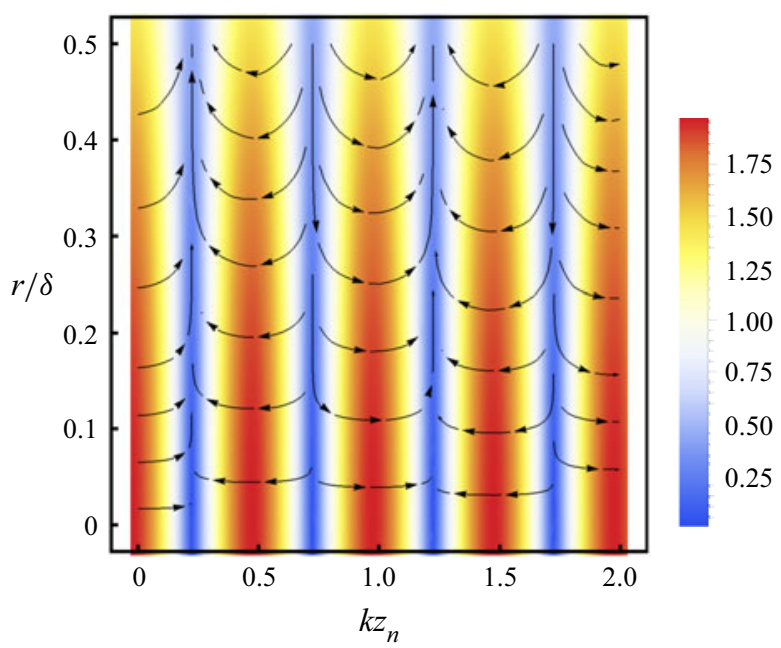

Figure 4. Spatial variations of the velocity field, given by the streamfunction $\psi_{0} / \delta^{2} U \cos \left(\theta_{n}\right)$, along the axial and radial coordinates $k z_{n}$ and $r / \delta$, respectively, where we ignore wave attenuation $\left(\alpha_{n}=0\right)$ and time, define the positions of the centre and surface of the pore at $r=0$ and $\delta / 2$, respectively, and represent the different properties using dimensional notation. We further use arrows to give the path of the velocity field and colours to indicate the relative flow intensity, which is quantified in the colour legend to the right.

(3.20) yields the solution to the problem

$$
\psi_{0}=\operatorname{Real}\left\{\frac{r I_{0}\left[(1+i) R_{0}\right]-(1-i) I_{1}[(1+i) r]}{2 I_{2}\left[(1+i) R_{0}\right]} \cos \left(\theta_{n}\right) r \mathrm{e}^{i\left(t-z_{n}\right)-\alpha_{n} z_{n}}\right\}
$$

where $I_{i}[Z]$ is the modified Bessel function of the first kind of order $i$; the argument $Z$ is an arbitrary complex number. While the solution in (3.21) is elegant, it is difficult to work with the complex Bessel functions. Hence, in preparation for calculating $\psi_{1}$, which will involve nonlinear operations, we substitute the modified Bessel functions by the series $I_{i}[Z]=\sum_{n=0}^{\infty}(\Gamma(n+i+1) n !)^{-1}(Z / 2)^{2 n+i}$, where $\Gamma()$ is the gamma function. For the positive and integer sum $n+i$, it may be simplified to $\Gamma(n+i+1)=(n+i)$ !. Employing the two first terms in the series for the modified Bessel functions in (3.21) gives that

$$
\psi_{0} \approx \frac{3 \cos \left(\theta_{n}\right) r^{2}\left(2 R_{0}^{2}-r^{2}\right)\left(R_{0}^{2} \sin \left(t-z_{n}\right)+6 \cos \left(t-z_{n}\right)\right)}{R_{0}^{2}\left(R_{0}^{4}+36\right)} \mathrm{e}^{-\alpha_{n} z_{n}}
$$

which differs from the exact solution in (3.21) by up to $2.7 \%$ for $R_{0} \leq 1$ (corresponding to $D_{p} \leq 2 \delta$ in dimensional terms). We illustrate the streamlines of the flow field in figure 4. The leading-order volume flux in the pore is periodic and hence will vanish over long times. Next, we calculate the steady component of the second-order problem which becomes the leading-order measurable, component of the flow at long times. 


\section{Acoustic flow in porous media}

\subsubsection{Steady second-order problem}

We use the time averaging operator \langle\rangle to find that the steady state component of the order of magnitude $O\left(S t^{-1}\right)$ problem is given by

$$
\begin{gathered}
-3 \frac{\langle\psi\rangle_{1, r}}{r^{3}}+3 \frac{\langle\psi\rangle_{1, r r}}{r^{2}}-2 \frac{\langle\psi\rangle_{1, r r r}}{r}+\langle\psi\rangle_{1, r r r r}=\text { forcing. } \\
\text { forcing } \equiv\left\langle 3 \frac{\psi_{0, z_{n}} \psi_{0, r}}{r^{3}}+\frac{\psi_{0, r} \psi_{0, r z_{n}}}{r^{2}}-3 \frac{\psi_{0, z_{n}} \psi_{0, r r}}{r^{2}}-\frac{\psi_{0, r} \psi_{r r z_{n}}}{r}+\frac{\psi_{0, z_{n}} \psi_{0, r r r}}{r}\right\rangle, \\
\left.\langle u\rangle_{1}\right|_{r=R_{0}}=-\left.\left\langle u_{0, r} R_{1}\right\rangle\right|_{r=R_{0}}, \quad \partial\langle u\rangle_{1} /\left.\partial r\right|_{r=0}=\left.\langle v\rangle_{1}\right|_{r=0}=0,
\end{gathered}
$$

where the condition on the volume flux is omitted since it is not known; as noted before, steady volume flux in the pores is our goal in this analysis. The additional constraint required to solve (3.23) is the absence of a steady pressure distribution in the pore. Moreover, the no-slip condition for the flow at the pore surface in (3.23) is found by expanding the velocity field in the radius of pore, $R$, about its magnitude at rest, $R_{0}$, which gives $\left.u\right|_{r=R}=\left.u\right|_{r=R_{0}}+\left.u_{r}\right|_{r=R_{0}} S t^{-1} R_{1}+\cdots=0$. Further accounting for the expansion of the axial velocity, $u$, in (3.19) gives that $\left.u_{0}\right|_{r=R_{0}}+\left.S t^{-1}\left(u_{1}+u_{0, r} R_{1}\right)\right|_{r=R_{0}}+\cdots=0$. The property, $R_{1}$, is given from the kinematic condition in (3.18). The latter translates to $R_{1, t}=\left.v_{0}\right|_{r=R_{0}}+\cdots$ subject to the series expansion in (3.19). Averaging the result over long times, collecting terms of the order of magnitude $O\left(S t^{-1}\right)$ and using the result for $\psi_{0}$ in (3.22) gives the boundary condition

$$
\left.\langle u\rangle_{1}\right|_{r=R}=-\left.\left\langle u_{0, r} R_{1}\right\rangle\right|_{r=R_{0}}=\frac{36 \cos ^{2}\left(\theta_{n}\right)}{36+R_{0}^{4}} \mathrm{e}^{-2 \alpha_{n} z_{n}} .
$$

The forcing term in (3.23) is given by

$$
\text { forcing }=\frac{144 r^{2}\left(r^{2}-R_{0}^{2}\right)}{R_{0}{ }^{4}\left(R_{0}{ }^{4}+36\right)} \alpha_{n} \cos ^{2}\left(\theta_{n}\right) \mathrm{e}^{-2 \alpha_{n} z_{n}},
$$

when using the result for $\psi_{0}$ in (3.22). The problem in (3.23), subject to the result for the forcing term in (3.25), is satisfied by the general solution

$$
\begin{aligned}
\langle\psi\rangle_{1}= & c_{1}\left(z_{n}\right) r^{2}+c_{2}\left(z_{n}\right)(-2+4 \log (r)) r^{2}+c_{3}\left(z_{n}\right) r^{4}+c_{4}\left(z_{n}\right) \\
& -\frac{r^{6}\left(6 R_{0}{ }^{2}-r^{2}\right)}{8 R_{0}{ }^{4}\left(R_{0}{ }^{4}+36\right)} \alpha_{n} \cos ^{2}\left(\theta_{n}\right) \mathrm{e}^{-2 \alpha_{n} z_{n}},
\end{aligned}
$$

where $c_{1}\left(z_{n}\right), c_{2}\left(z_{n}\right), c_{3}\left(z_{n}\right), c_{4}\left(z_{n}\right)$ are coefficients of integration. The coefficient $c_{3}\left(z_{n}\right)$ is associated with a steady pressure distribution along the pore. The absence of a pressure distribution renders $c_{3}\left(z_{n}\right)=0$. Further accounting for the boundary condition in (3.24) and the requirement that the velocity field is symmetrical about the centre of the pore in (3.23) gives that

$$
\langle\psi\rangle_{1}=\frac{144 R_{0}^{4}+\alpha_{n}\left(r^{6}-6 r^{4} R_{0}^{2}+14 R_{0}^{6}\right)}{8 R_{0}^{4}\left(R_{0}^{4}+36\right)} \cos ^{2}\left(\theta_{n}\right) r^{2} \mathrm{e}^{-2 \alpha_{n} z_{n}} .
$$

We show the distribution of the axial velocity along the pore for $R_{0} \equiv D_{p} / 2$ and for different values of the attenuation coefficient of the acoustic wave, $\alpha_{n}$, in figure 5 . Increasing the magnitude of $\alpha_{n}$ increases the steady acoustic flow velocity in the pore. 


\section{O. Manor}

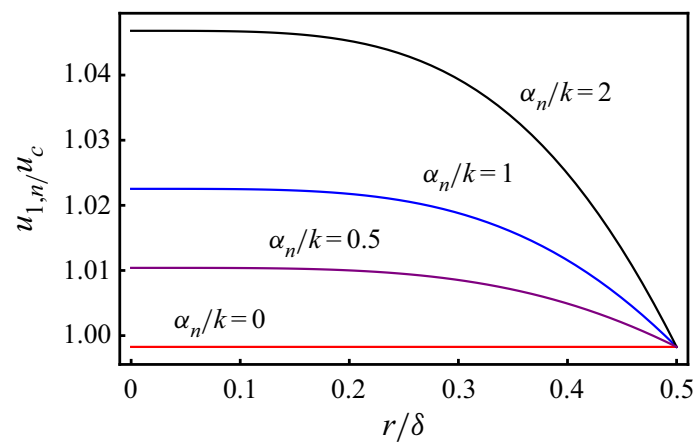

Figure 5. Radial $(r)$ variations of the dimensional axial drift velocity, $u_{n}$, along pore $n$ for different values of the effective wave attenuation coefficient along the pore, where we use dimensional notation and $u_{c} \equiv$ $S t^{-1} \cos ^{2}\left(\theta_{n}\right) \mathrm{e}^{-2 \alpha_{n} z n} U \alpha_{n}$.

The volume flux along the pore is given by $Q=2 \pi\left(\left.\langle\psi\rangle\right|_{r=R_{0}}-\left.\langle\psi\rangle\right|_{r=0}\right)$ and the corresponding spatially averaged drift velocity along the pore is

$$
u_{\text {pore }, n}=\frac{Q}{\langle A\rangle}=S t^{-1} \frac{9 \cos ^{2}(\theta)}{4} \frac{16+\alpha_{n} R_{0}^{2}}{36+R_{0}{ }^{2}} \mathrm{e}^{-2 \alpha_{n} z_{n}}
$$

where $\langle A\rangle \approx \pi R_{0}^{2}$ is the time averaged radial cross-sectional area of the pore. The average velocity field along the acoustic path in the integral porous medium is then given by

$$
\mathcal{U}=\frac{\zeta}{N} \sum_{n=1}^{N} \cos \left(\theta_{n}\right) u_{\text {pore }, n}=\frac{9 \zeta S t^{-1}}{4} \frac{16 / m^{\prime}+\alpha R_{0}^{2} / m}{36+R_{0}^{4}} \mathrm{e}^{-2 \alpha x},
$$

noting again that $\alpha=\alpha_{n} / \cos \left(\theta_{n}\right)$ and $\alpha_{n} z_{n}=\alpha x$. Using dimensional terms, this result reads,

$$
\mathcal{U}=\frac{9 \zeta S t^{-1} U}{4} \frac{16 / m^{\prime}+\alpha R_{0}^{2} / m \delta}{36+\left(R_{0} / \delta\right)^{4}} \mathrm{e}^{-2 \alpha x}=\frac{9 \zeta S t^{-1} U}{4} \frac{16 / m^{\prime}+\alpha D_{p}^{2} / 4 m \delta}{36+\left(D_{p} / 2 \delta\right)^{4}} \mathrm{e}^{-2 \alpha x},
$$

where we employed the definition $R_{0} \equiv D_{p} / 2$ following the second equality. The expression in (3.30) is associated with the sum of a term which originates from Rayleigh streaming and a term which originates from Eckart streaming. The latter is multiplied by the attenuation coefficient of the acoustic wave, $\alpha$. In the limit of small pores, where $D_{p} / \delta \ll 1$, this expression further simplifies to

$$
\mathcal{U} \approx S t^{-1} \zeta U \mathrm{e}^{-2 \alpha x} / m^{\prime}
$$

The Eckart streaming-type contribution to the flow vanishes and the contribution to the flow appears solely from a Rayleigh streaming-type contribution.

\section{Discussion}

It is of value to compare the steady acoustic flow in the different results given in (2.6), where the porous medium and fluid possess the same acoustic properties, and in (3.15), (3.30) and (3.31), where the porous medium has rigid and large, medium to small and small pores, respectively. In particular, for different flow regimes, it is instructive to assess the difference between the steady acoustic flow, i.e. the acoustic streaming, subject to 


\section{Acoustic flow in porous media}

different properties of the porous medium. We show that (2.6) appears from a similarity between the Darcy equation for flow through porous media under a pressure gradient and the equations that govern steady acoustic flow in porous media in the specific case of the same acoustic properties in the solid and fluid therein. This type of analysis is not trivial in the case of rigid porous media. However, one may employ the similarity between the result in (2.6) and the later results for rigid porous media to identify approximate permeability coefficients for each case.

One may write the result for large pores in (3.15) in a similar manner to the Darcy equation-type formula in (2.6) by identifying two quasi-permeability coefficients. For convenience, we take a different approach to writing quasi-Darcy formulas, which is intuitive for acoustic flow. Instead of writing permeability coefficients multiplied by the force per unit volume in porous media, as is the Darcy equation, we suggest quasi-permeability coefficients whose product with the local particle velocity of the acoustic wave, $U \mathrm{e}^{-2 \alpha x}$ will give the effective flow through the porous medium in the form: $\mathcal{U}=\left(K^{\prime} \times R e+K^{\prime \prime} \times S t^{-1}\right) \times U \mathrm{e}^{-2 \alpha x}$. The first term between the brackets is associated with acoustic forcing in the bulk of the pores (Eckart streaming). The second term is associated with the interaction between the acoustic wave and the pore surface, which results in an acoustic boundary layer flow (Rayleigh streaming). The corresponding quasi-permeability coefficients are $K^{\prime} \equiv \zeta \alpha D_{p} / 32 m$ and $K^{\prime \prime} \equiv 3 \zeta / 4 m^{\prime}$. A similar analysis to the above is appropriate for the case of medium to small pores in (3.30). Here, the permeability coefficients are $K^{\prime}=\left(9 \zeta \alpha k / 8 m \delta^{3}\right) \times\left(1 /\left(36+\left(D_{p} / 2 \delta\right)^{4}\right)\right)$ and $K^{\prime \prime} \equiv\left(36 \zeta / m^{\prime}\right) /\left[36+\left(D_{p} / 2 \delta\right)^{4}\right]$. In the limit of small pores in (3.31), the driving mechanism for the flow is solely Rayleigh streaming. Hence, one may employ just one permeability coefficient, $K^{\prime}=0, K^{\prime \prime} \equiv \zeta / m^{\prime}$. The corresponding effective flow through the porous medium is given by $\mathcal{U}=K^{\prime \prime} \times S t^{-1} U \mathrm{e}^{-2 \alpha x}$. Thus, one may use experiment to measure the coefficient $K^{\prime \prime \prime}=K^{\prime} \times R e+K^{\prime \prime} \times S t^{-1}$ for rigid porous media to estimate the magnitude of a steady acoustic flow therein. A quantitative comparison between the different equations derived for rigid porous media is given next.

In figure 6 , we plot the different results for the scaled steady acoustic flow, $\mathcal{U}$, in rigid porous media for various pore sizes, $D_{p}$, and a random pore azimuth distribution $(m=$ $\left.3, m^{\prime}=4\right)$. For the case of large pores in (3.15), we plot the equation $\mathcal{U} / U \zeta S t^{-1} \mathrm{e}^{-2 \alpha x} \approx$ $D_{p} \alpha \operatorname{ReSt} / 96+3 / 16=\left(D_{p} / \delta\right)^{2}(\alpha / k) / 48+3 / 16$ (applicable for $\left.D_{p} / \delta \gg 1\right)$. For the case of medium to small pores in (3.30), we plot the equation $\mathcal{U} / U \zeta S t^{-1} \mathrm{e}^{-2 \alpha x} \approx(9 / 4) \times$ $\left[4+\left(D_{p} / \delta\right)^{2}(\alpha \delta) / 12\right] /\left[36+\left(D_{p} / \delta\right)^{4} / 16\right]$ (applicable for $\left.D_{p} / \delta<2\right)$. For the case of the limit of small pores in (3.31), we plot the equation $\mathcal{U} / U \zeta S t^{-1} \mathrm{e}^{-2 \alpha x} \approx 1 / 4$ (applicable for $\left.D_{p} / \delta \ll 1\right)$. It is possible to show that both $\alpha / k$ and $\alpha \delta$ are small numbers in the corresponding equations. Hence, we allocate these quantities values between 0 to 1 in the figure.

The curves for the steady acoustic flow, $\mathcal{U}$, that are products of the limit of small pores and of the case of medium to small pores, coincide as $D_{p} / \delta \rightarrow 0$. The limit of small pores is independent of the ratios $D_{p} / \delta, \alpha \delta$ and $\alpha / k$. In the other cases, we observe an increase in the magnitude of the scaled $\mathcal{U}$ when increasing the magnitudes of $D_{p} / \delta, \alpha \delta$ and $\alpha / k$, at least within the pore size applicability regions of the equations. In particular, the trend of an increase in the scaled $\mathcal{U}$ when increasing $D_{p} / \delta$ is reversed in a non-physical manner at approximately $D_{p} / \delta \gtrsim 2$ in the case of (3.30). The non-physical behaviour originates from the truncation of the Bessel series during the derivation of (3.30) and was noted above. It is clear then that one must introduce more terms in the truncated Bessel series to obtain realistic results for $D_{p} / \delta>2$ in this case. Moreover, it is interesting to note that the result 


\section{O. Manor}
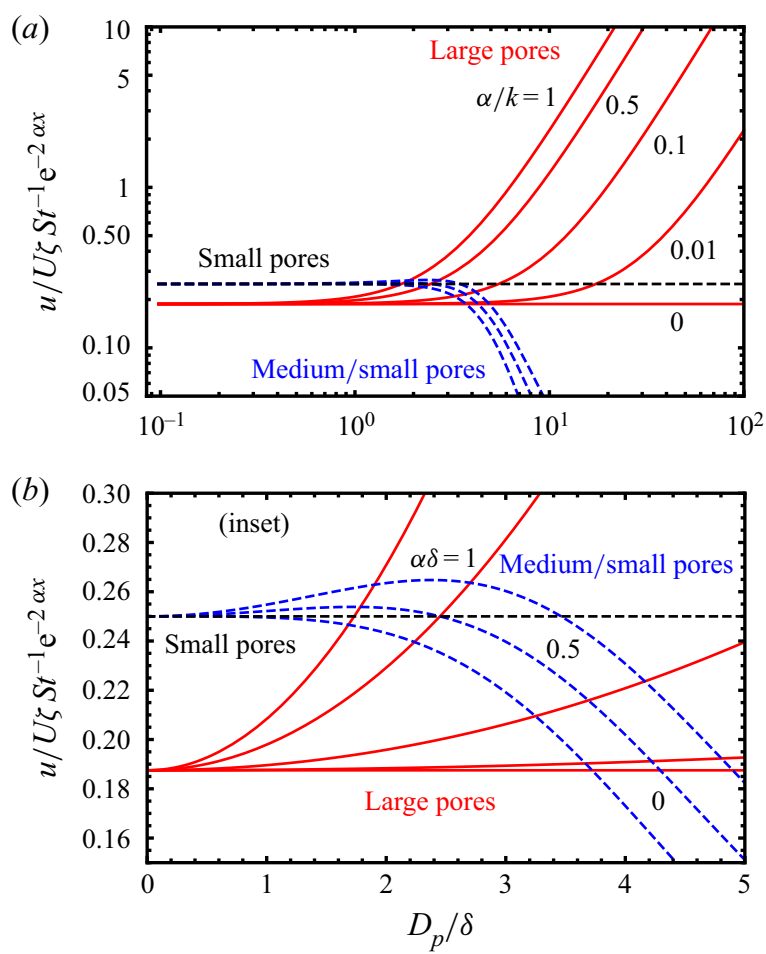

Figure 6. Scaled pore size, $D_{p} / \delta$, variations of the scaled steady acoustic flow, $\mathcal{U} / U \zeta S t^{-1} \mathrm{e}^{-2 \alpha x}$, in rigid porous media of randomly distributed pore azimuths with respect to the acoustic path $\left(m=3, m^{\prime}=4\right)$ for the cases of small pores in (3.31) (dashed black line), medium to small pores in (3.30) (dashed blue line) for $\alpha \delta=0,0.5,1$ and large pores (red solid lines) in (3.15) for $\alpha / k=0,0.01,0.1,0.5$, 1 , where $(b)$ is an inset of panel (a) for small pore sizes and where (3.31) is applicable for $D_{p} / \delta \ll 1$, (3.30) is applicable for $D_{p} / \delta<2$ and (3.15) is applicable for $D_{p} / \delta \gg 1$.

for the scaled $\mathcal{U}$, subject to large pores in (3.15), is not too different from the exact result for small pores in (3.31) for a vanishing pore size, $D_{p} / \delta \rightarrow 0$. In particular, the result by the latter equation is $1 / 4$. The result by the former equation is $3 / 16$. The ratio between the two results is $3 / 4$. As a reminder, the main constraint employed when deriving (3.15) is that we ignore the pore curvature when calculating the acoustic boundary layer flow (Rayleigh streaming). Hence, the cost of this geometrical simplification to the magnitude of $\mathcal{U}$ outside the applicability region of (3.15) appears to be small, at least in this case, considering the small deviation of (3.15) from (3.31) for $D_{p} / \delta \rightarrow 0$.

\section{Conclusions and summary}

In this study, we calculate the steady acoustic flow, also known as acoustic streaming or drift, along the path of an acoustic wave in a solid porous medium. The common denominator in our findings is that the propagation of an acoustic wave in a porous medium supports an effective acoustic flow. For same acoustic properties in the fluid and solid, the acoustic forcing for flow is equivalent to a pressure gradient throughout the porous medium. The similarity to the Darcy equation for flow in porous media yields an expression for the acoustic flow, which is proportional to the square of the particle velocity and attenuation coefficient of the acoustic wave, $U$ and $\alpha$, the Darcy permeability and the reciprocal of the structural coefficient of the solid medium, $K$ and $m$, respectively, and 


\section{Acoustic flow in porous media}

the reciprocal of the shear viscosity of the fluid therein, $\mu$. In the general case where the acoustic properties of the fluid deviate from those of the solid, the acoustic forcing for flow is further associated with surface effects. The similarity to the Darcy equation is not trivial in this case. The effective velocity of the acoustic flow is found in the rigid porous frame limit to be proportional to the particle velocity and attenuation coefficient of the acoustic wave, $U$ and $\alpha$, the porosity of the medium, $\zeta$, the reciprocals of the two structural factors $m$ and $m^{\prime}$, the reciprocal of the Strouhal number, $S t^{-1}=U k / \omega$, and the Reynolds number in the pores, $\operatorname{Re}=\rho U D_{p} / \mu$, where $k, \omega, D_{p}$ and $\rho$ are the acoustic wavenumber and angular frequency, the diameter of the pores (assumed cylindrical) and the density of liquid, respectively. Below, we briefly summarize and discuss our assumptions and results.

In our analysis, we assume that the acoustic wavelength is large compared with the characteristic size of the pores. Under this condition, the acoustic wave, which propagates through a porous medium, may be approximated in the form of a planar acoustic wave, such as sound or ultrasound waves, (2.1),

$$
u_{\text {wave }}=U \cos (\omega t-k x) \mathrm{e}^{-\alpha x},
$$

where $t$ and $x$ are time and the axial coordinate along the path of the wave, respectively.

When the acoustic properties of the fluid and solid are the same, the solid/fluid interface is 'transparent' to the acoustic wave and both phases support similar particle velocities along the path of the wave. A consequence of our assumptions is that the acoustic flow in the pore is solely a product of the Eckart streaming. It is generated by the inertial transport of momentum from the attenuating acoustic wave to flow. The steady velocity of the acoustic flow in this case is given by (2.6),

$$
\mathcal{U}=\frac{2 \rho \alpha K U^{2}}{m \mu} \mathrm{e}^{-2 \alpha x},
$$

where the structural coefficient, $m$, is a function of the average azimuth between the axial coordinates of the pores and the acoustic path. It accepts values of 1,3 and $\infty$ for the cases where the pores are aligned along, randomly and perpendicular to the path of the wave, respectively. Moreover, if one may postulate that the acoustic wave mostly attenuates by viscous dissipation in the fluid phase, one may approximate to leading order the attenuation coefficient by $\alpha \approx \mu b k^{3} \zeta / 2 \rho \omega$, where $\rho$ is the density of the fluid, $\mu b \equiv \mu_{b}+4 \mu / 3$ and $\mu_{b}$ is the bulk viscosity coefficient. This is a simple product of the wave attenuation coefficient in fluid (Nyborg 1965) and the porosity factor.

In a rigid porous frame, the acoustic wave is assumed to propagate solely along the fluid in the pores; the porous solid is static. The problem for the wavenumber and rate of acoustic wave attenuation under such conditions was solved previously and appears to be in good agreement with experiment (Zwikker \& Kosten 1949; Morse 1952). We consider three cases that are similar to well-known analytical results for the wave attenuation given in the literature: the first case is of a pore diameter that is large compared with the viscous penetration length of the acoustic wave, $D_{p} \gg \delta$, where $\delta \equiv \sqrt{2 \mu / \rho \omega}$. The second case is of a pore diameter that is comparable to or smaller than the viscous penetration length, $D_{p}<2 \delta$. The third case is the limit of small pores, $D_{p} \ll \delta$.

The steady acoustic flow in a pore is forced by two mechanisms, which transfer instantaneous momentum from the acoustic wave to steady flow. One mechanism dominates the bulk of the pore and is associated with the attenuation of the acoustic wave - Eckart streaming. The other mechanism dominates the vicinity of the pore surface - a Rayleigh-type streaming. We show that one may write corresponding quasi-Darcy equations for the different cases in the rigid porous frame in the form $\mathcal{U}=K^{\prime \prime \prime} U \mathrm{e}^{-2 \alpha x}$. 


\section{O. Manor}

The quasi-permeability coefficient, $K^{\prime \prime \prime}$, is a measurable quantity and a function of the dimensionless numbers $R e \equiv \rho U D_{p} / \mu$ and $S t^{-1} \equiv U k / \omega$.

The effective steady acoustic flow in a porous medium of large pores is given by (3.15),

$$
\mathcal{U} \approx U \zeta\left(\frac{D_{p} \alpha}{32} \frac{R e}{m}+\frac{3}{4} \frac{S t^{-1}}{m^{\prime}}\right) \mathrm{e}^{-2 \alpha x},
$$

where $m^{\prime}$ is a modified structural parameter. The latter accepts values of 1,4 and $\infty$ for the cases where the pores are aligned along, randomly and perpendicular to the path of the wave, respectively. Previous studies suggest that the rate of attenuation of the wave for this case may be approximated to leading order by $\alpha \approx(2 q)^{-1}\left(\delta k / D_{p}\right)$, where $q$ is the ratio between the pore size in the presence and absence of the acoustic wave. The effective steady acoustic flow in a porous medium of medium to small pores is given for $D_{p}<2 \delta$ by (3.30),

$$
\mathcal{U}=\frac{9 \zeta S t^{-1} U}{4} \frac{16 / m^{\prime}+\alpha D_{p}^{2} / 4 m \delta}{36+\left(D_{p} / 2 \delta\right)^{4}} \mathrm{e}^{-2 \alpha x} .
$$

Moreover, in the limit of small pores, $D_{p} \ll \delta$, the above result is simplified to (3.31),

$$
\mathcal{U} \approx S t^{-1} \zeta U \mathrm{e}^{-2 \alpha x} / m^{\prime}
$$

When considering a case study for randomly distributed pores and comparing the different results in the range $0<D_{p} / \delta<100$, we observe that the predictions for the steady acoustic flow, $\mathcal{U}$, in the limit of small pores in (3.31) and in the case of medium to small pores in (3.30) are similar for a vanishing pore size. The steady acoustic streaming in the limit of small pores is solely forced by a Rayleigh streaming-type mechanism. The steady acoustic flow in the case of medium to small pores is governed by contributions from both Rayleigh and Eckart streaming-type mechanisms. Increasing the size of the pores, (increasing $D_{p} / \delta$ values), also increases the steady acoustic flow, $\mathcal{U}$, at least for small $D_{p} / \delta$ values. Above a threshold value of $D_{p} / \delta$, we observe that $\mathcal{U}$ decreases in magnitude in an unphysical manner. This result emphasizes the limited applicability of the expression in (3.30) for $D_{p}<2 \delta$. To obtain realistic results for $D_{p}>2 \delta$ in the case of small to medium pores in (3.30), one must introduce additional terms in the truncated Bessel series when calculating (3.30). In the case of large pores in (3.15), we find that $\mathcal{U}$ increases in magnitude when increasing the size of pores (increasing $D_{p} / \delta$ values). Interestingly, (3.15) appears to give a reasonable prediction for $\mathcal{U}$ also in the limit of a vanishing pore size. The main constraint on the large pore result is that we ignore the pore curvature when calculating the acoustic boundary layer flow (Rayleigh streaming). Under the assumption of randomly distributed pores, we observe that the geometrical simplifications in (3.15) give a prediction that deviates from the exact limit for small pores in (3.31) by a factor of $3 / 4$ for a vanishing pore size $\left(D_{p} \rightarrow 0\right)$.

It is of value to note that the analysis which yields (2.6), (3.15), (3.30) and (3.31) is appropriate for the case where the pressure throughout the porous medium is the ambient pressure. This assumption corresponds to the case where the front width of the acoustic wave is smaller than the width of the porous medium and the pores are interconnected. In the case where this analysis is not relevant, one may consider an opposite case where the distribution of steady pressure in the porous medium is solely a function of the acoustic wave and fluid mass conservation. This case is appropriate when the acoustic front may be considered flat (its intensity is independent of the direction normal to the path of the wave), the acoustic front is at least as wide as the porous medium, the porous medium is 


\section{Acoustic flow in porous media}

closed to liquid flow on the sides and the pressure at the entrance to the porous medium is the ambient pressure. The conservation of fluid mass will result in a distribution of steady pressure in the porous medium. Overall, this opposite limit for the pressure distribution will result in similar expressions for the effective acoustic flow given, albeit in the absence of the decaying exponent term $\mathrm{e}^{-2 \alpha x}$. Moreover, in this case, the quasi-Darcy equation will take the form, $\mathcal{U}=K^{\prime \prime \prime} U$.

Finally, the characteristic frequency regime employed in the present literature on acoustofluidics is mostly in the range of $0.1-400 \mathrm{MHz}$. The given range of acoustic frequencies translates to a range of viscous penetration lengths of $\delta \approx 1400-20 \mathrm{~nm}$ in water at room temperature. Hence, by altering the realistic pore size, which traverse the characteristic lengths of 1 nanometre to 1 millimetre, and the given acoustic frequency range, one may traverse in the laboratory the regimes of $D_{p}<\delta$ and $D_{p} \gg \delta$. Moreover, there are several cases which are not considered in this analysis and may deserve future work. These include acoustic streaming when the acoustic wave propagates solely through the solid, through both the solid and fluid, varies in time and when the acoustic wavelength is small (or comparable) to the pore size. Other cases of interest are acoustic streaming in porous media that include a distribution of pore sizes and in deformable porous media, where the pores greatly deform in the presence of acoustic waves and flow.

Funding. We acknowledge support of this research by the Israel Science Foundation (ISF) under grant number $441 / 20$.

Declaration of interest. The authors report no conflict of interest.

\section{Author ORCIDs.}

(D) Ofer Manor https://orcid.org/0000-0003-1526-5266.

\section{REFERENCES}

Altshuler, G. \& MAnOR, O. 2015 Spreading dynamics of a partially wetting water film atop a MHz substrate vibration. Phys. Fluids 27 (10), 102103.

Altshuler, G. \& MANOR, O. 2016 Free films of a partially wetting liquid under the influence of a propagating MHz surface acoustic wave. Phys. Fluids 28 (7), 072102.

AnG, K.M., YeO, L.Y., Hung, Y.M. \& TAN, M.K. 2017 Acoustially-mediated microfluidic nanofiltration through graphene films. Nanoscale 9, 6497-6508.

ArzT, R.M. \& SAlzmann, E. 1967 Elastic surface waves in quartz at $316 \mathrm{MHz}$. Appl. Phys. Lett. 10 (5), $165-167$.

Attenborough, K. 1971 The influence of microstructure on propagation in porous fibrous absorbents. J. Sound Vib. 16, 419-442.

Attenborough, K. 1982 Acoustical characetristics of porous materials . Phys. Rep. 82, 179-227.

BERANEK, L.L. 1947 Acoustical properties of homogeneous, isotropic rigid tiles and flexible blankets. J. Acoust. Soc. Am. 19 (4), 556-568.

Biot, M.A. 1962 Generalized theory of acoustic propagation in porous dissipative media. J. Acoust. Soc. Am. 34 (9A), 1254-1264.

BUCKINGHAM, M.J. 1997 Theory of acoustic attenuation, dispersion, and pulse propagation in unconsolidated granular materials including marine sediments. J. Acoust. Soc. Am. 102 (5), 2579-2596.

CAMPBELl, J.J. 1970 Propagation of surface waves at the boundary between a piezoelectric crystal and a fluid medium. IEEE Trans. Sonics Ultrasonics 2, 71-76.

CARMAN, P.C. 1937 Fluid flow through granular beds. Trans. Inst. Chem. Engng 15, 150-166.

CARMAN, P.C. 1956 Flow of Gases through Porous Media. Butterworths.

Chladni, E.F.F. 1787 Entdeckungen uber die Theory des Klanges. Breitkopf und Hartel.

Connacher, W., Zhang, N., Huang, A., Mei, J., Zhang, S., Gopesh, T. \& Friend, J.R. 2018 Micro/nano acoustofluidics: materials, phenomena, design, devices, and applications. Lab on a Chip 18, 1952-1996.

DARCY, H. 1856 Les fontaines publiques de la ville de Dijon. Dalmont. 


\section{O. Manor}

Doinikov, A.A., Thibault, P. \& Marmottant, P. 2017 Acoustic streaming in a microfluidic channel with a reflector: case of a standing wave generated by two counterpropagating leaky surface waves. Phys. Rev. E 96 (1), 013101.

ECKART, C. 1948 Vortices and streams caused by sound waves. Phys. Rev. 73 (1), 68.

Elkhoury, J.E., Brodsky, E.E. \& AGnew, D.C. 2006 Seismic waves increase permeability. Nature 441 (7097), 1135-1138.

ERGun, S. 1952 Fluid flow through packed columns. Chem. Engng Prog. 48, 89-94.

FARADAY, M. 1831 On a peculiar class of acoustical figures; and on certain forms assumed by groups of particles upon vibrating elastic surfaces. Phil. Trans. R. Soc. Lond. 121, 299-340.

FRIEND, J.R. \& YEO, L. 2011 Microscale acoustofluidics: microfluidics driven via acoustics and ultrasonics. Rev. Mod. Phys. 83 (2), 647-704.

Guttenberg, Z., Rathgeber, A., Keller, S., Radler, J.O., Wixforth, A., Kostur, M., SChindler, M. \& TAlkner, P. 2004 Flow profiling of a surface-acoustic-wave nanopump. Phys. Rev. E 70, 056311 .

Haire, T.J. \& LAngton, C.M. 1999 Biot theory: a review of its application to ultrasound propagation through cancellous bone. Bone 24 (4), 291-295.

HAMIDA, T. \& BABADAGLI, T. 2008 Displacement of oil by different interfacial tension fluids under ultrasonic waves. Colloids Surf. A 316 (1-3), 176-189.

Hamilton, M.F., Ilinskit, Y.A. \& ZabolotskayA, E.A. 2003 Acoustic streaming generated by standing waves in two-dimensional channels of arbitrary width. J. Acoust. Soc. Am. 113 (1), 153-160.

Ho, J., Tan, M.K., Go, D.B., Yeo, L.Y., Friend, J.R. \& Chang, H.-C. 2011 Paper-based microfluidic surface acoustic wave sample delivery and ionization source for rapid and sensitive ambient mass spectrometry. Anal. Chem. 83 (9), 3260-3266.

Horesh, A., Khaikin, D., Karnilaw, M., Zigelman, A. \& Manor, O. 2019 Acoustogravitational balance in climbing films. Phys. Rev. Fluids 4, 022001.

Horesh, A., Morozov, M. \& Manor, O. 2017 Enhanced drainage and thinning of liquid films between bubbles and solids that support surface waves. Phys. Rev. E 95 (5), 052803.

Horesh, A., Zigelman, A. \& MAnOR, O. 2020 Stabilizing water films using surface acoustic waves. Phys. Rev. Fluids 5, 114002.

Hosokawa, A. \& Otani, T. 1997 Ultrasonic wave propagation in bovine cancellous bone. J. Acoust. Soc. Am. 101 (1), 558-562.

Johnson, D.L. 2001 Theory of frequency dependent acoustics in patchy-saturated porous media. J. Acoust. Soc. Am. 110 (2), 682-694.

Kenneth, D.F., Keith, M. \& Shawn, M. 2004 Acoustic streaming in micro-scale cylindrical channels. Appl. Acoust. 65 (11), 1121-1129.

KirchHofF, G. 1868 Ueber den einfluss der wärmeleitung in einem gase auf die schallbewegung. Ann. Phys. 210 (6), 177-193. Available at: https://onlinelibrary.wiley.com/doi/pdf/10.1002/andp.18682100602.

KoZNEY, J. 1927 Ueber kapillare leitung des wassers im boden. Sitz.ber. Akad. Wiss. Wien 136, $271-306$.

LEAL, L.G. 2007 Advanced Transport Phenomena: Fluid Mechanics and Convective Transport Processes. Cambridge University Press.

Lighthill, J. 1978 Acoustic streaming. J. Sound Vib. 61, 391-418.

Lo, W.C., Sposito, G. \& MAJER, E. 2005 Wave propagation through elastic porous media containing two immiscible fluids. Water Resour. Res. 41 (2), W02025.

Longuet-Higgins, M.S. 1953 Mass transport in water waves. Phil. Trans. R. Soc. Lond. 245, 535-581.

MANOR, O., REZK, A.R., FriEnd, J.R. \& YEO, L.Y. 2015 Dynamics of liquid films exposed to high-frequency surface vibration. Phys. Rev. E 91, 053015.

MANOR, O., YEO, L.Y. \& FRIEND, J.R. 2012 The appearance of boundary layers and drift flows due to high-frequency surface waves. J. Fluid Mech. 707, 482-495.

Martinez, A.W., Phillips, S.T., Whitesides, G.M. \& Carrilho, E. 2010 Diagnostics for the developing world: microfluidic paper-based analytical devices. Anal. Chem. 82 (1), 3-10.

MiAnSARI, M. \& FRIEND, J.R. 2016 Acoustic nanofluidics via room-temperature lithium niobate bonding: a platform for actuation and manipulation of nanoconfined fluids and particles. Adv. Funct. Mater. 26 (43), 7861-7872.

Morozov, M. \& Manor, O. 2016 An extended Landau-Levich model for the dragging of a thin liquid film with a propagating surface acoustic wave. J Fluid Mech. 810, 307-322.

Morse, R.W. 1952 Acoustic propagation in granular media. J. Acoust. Soc. Am. 24 (6), 696-700.

Morse, P.M. \& Bolt, H.R. 1944 Sound waves in rooms. Rev. Mod. Phys. 16, 69.

Nyborg, W.L. 1952 Acoustic streaming due to attenuated plane waves. J. Acoust. Soc. Am. 25, 1-8.

Nyborg, W.L.M. 1965 Acoustic streaming. Phys. Acoust. 2, 265-331. 


\section{Acoustic flow in porous media}

Olny, X. \& Boutin, C. 2003 Acoustic wave propagation in double porosity media. J. Acoust. Soc. Am. $114(1), 73-89$.

Parolo, C. \& Merkoçı, A. 2013 Paper-based nanobiosensors for diagnostics. Chem. Soc. Rev. 42 (2), $450-457$.

Pavlic, A. \& DuAl, J. 2021 On the streaming in a microfluidic Kundt's tube. J Fluid Mech. 911, A28.

Poesio, P., OOMs, G., BARAKe, S. \& VAN DER BAS, F. 2002 An investigation of the influence of acoustic waves on the liquid flow through a porous material. J. Acoust. Soc. Am. 111 (5), 2019.

PRIDE, S.R. \& BERRYMAN, J.G. 2003 Linear dynamics of double-porosity dual-permeability materials. I. Governing equations and acoustic attenuation. Phys. Rev. E 68 (3,2), 036603.

Pride, S.R., Morgan, F.D. \& Gangi, A.F. 1993 Drag forces of porous-medium acoustics. Phys. Rev. B 47 (9), 4964-4978.

RAGHAVAN, R. 2018 Theory for acoustic streaming in soft porous matter and its applications to ultrasound-enhanced convective delivery. J. Therapuetic Ultrasound 6, 6.

RAYLEIGH, LORD 1884 On the circulation of air observed in Kundt's tubes and on some allied acoustical problems. Phil. Trans. R. Soc. Lond. 175, 1-21.

RAYLEIGH, LORD 1945 Theory of Sound, vol. 2. Dover Publications, Inc.

Reyta, I., BAilliet, H. \& VAliere, J.C. 2014 Experimental investigation of acoustic streaming in a cylindrical wave guide up to high streaming reynolds numbers. J. Acoust. Soc. Am. 135, 27.

RezK, A.R., Manor, O., Friend, J.R. \& YeO, L.Y. 2012 Unique fingering instabilities and soliton-like wave propagation in thin acoustowetting films. Nat. Commun. 3, 1167.

RezK, A.R., Manor, O., Yeo, L.Y. \& Friend, J.R. 2014 Double flow reversal in thin liquid films driven by megahertz-order surface vibration. Proc. R. Soc. Lond. Ser. A 470, 20130765.

Riley, N. 1998 Acoustic streaming. Theor. Comput. Fluid Dyn. 10, 349-356.

Riley, N. 2001 Steady streaming. Ann. Rev. Fluid Mech. 33, 43-65.

Schlichting, H. 1932 Calculation of even periodic barrier currents. Phys. Z 33, 327-335.

ScotT, R.A. 1946 The absorption of sound in a homogeneous porous medium. Proc. Phys. Soc. 58 (2), $165-183$.

Shiokawa, S., MAtsui, Y. \& UEDA, T. 1989 Liquid streaming and droplet formation caused by leaky Rayleigh waves. In Proceedings, IEEE Ultrasonics Symposium 1989, vol. 1, pp. 643-646. IEEE.

SIDES, D.J. 2003 Application of a generalized acoustic propagation theory to fibrous absorbents. J. Sound Vib. 19, 49-64.

StUART, J.T. 1966 Double boundary layers in oscillatory viscous flow. J. Fluid Mech. 24, 673-687.

Westervelt, P.J. 2004 The theory of steady rotational flow generated by a sound field. J. Acoust. Soc. Am. $25,60-67$.

Williams, K.L., Jackson, D.R., Thorsos, E.I., TAng, D.J. \& Schock, S.G. 2002 Comparison of sound speed and attenuation measured in a sandy sediment to predictions based on the Biot theory of porous media. IEEE J. Ocean. Engng 27 (3), 413-428.

XU, Y. 2018 Nanofluidics: a new arena for materials science. Adv. Mater. 30 (3), 1702419.

YAO, Y., ZHANG, W., YANG, K., LIU, S. \& HE, B. 2012 Theoretical model on the heat and mass transfer in silica gel packed beds during the regeneration assisted by high-intensity ultrasound. Intl J. Heat Mass Transfer 55 (23-24), 7133-7143.

ZAREK, J.H.B. 2002 Sound absorption in flexible porous materials. J. Sound Vib. 61, 205-234.

ZWikker, C. \& Kosten, W. 1949 Sound Absorbing Materials. Elsevier Publishing Company Inc. 